\title{
An Autoregressive Doppler Spread Estimator for Fading Channels
}

\author{
Pengda Huang, Student Member, IEEE, Dinesh Rajan, Senior Member, IEEE, Joseph Camp, Member, IEEE
}

\begin{abstract}
Doppler spread causes a significant performance degradation in fast-fading mobile communication systems. This paper proposes a novel Doppler estimation scheme that uses a damped sinusoid to approximate the autocorrelation of the fading channel. Subsequently, an Autoregressive (AR) model is used for the damped sinusoid and the parameters of the model are related to the Doppler value. The superiority of the proposed estimation method over existing state-of-the-art methods is demonstrated using numerical simulations. The proposed scheme is promising in vehicular communication systems.
\end{abstract}

Index Terms-Doppler spread estimation, OFDM system, fading channel.

\section{INTRODUCTION}

$\mathbf{I}$ $\mathrm{N}$ a multipath fading channel, knowledge of the Doppler spread is useful to compensate and improve the link layer performance. Several methods have been proposed to acquire the Doppler information including a maximum likelihood estimator [1], an estimator that uses the cyclostationarity of the transmitted symbols [2], and an estimator that is based on the Fourier transform of the autocorrelation of the channel [3]. Various Doppler spread estimators are reviewed in [4], among which the level crossing rate (LCR) method and the covariance based method are emphasized. The LCR based Doppler estimator is also discussed in [5] and has low complexity. However, the LCR based approach is vulnerable to the additive noise. The covariance based method finds the Doppler value by minimizing the difference between the Bessel $(1,0)$ function and ACF of the fading channel [6].

Baddour and Beaulieu [7] studied the feasibility of representing a fading channel with an autoregressive model. A 2step strategy to calculate the Doppler is proposed in [8]. This method uses the AR models for the Rayleigh fading channel and a subsequent Kalman filter to calculate the Doppler. A Doppler spread estimator that is robust to carrier frequency offset is proposed in [9]. However, the estimator relies on the assumption that the power spectrum density (PSD) function of the fading channel is strictly symmetric. The coherence time of channel fading is used to estimate the Doppler [10]. However, the estimation method makes the assumption that autocorrelation function (ACF) equals half of maximum value at exactly half of the max-to-zero time length. Thus, such a Doppler estimation method will incur severely degraded performance when the channel ACF is distorted.

In this paper, we derive a new method to estimate the maximum Doppler spread in a fading channel. The proposed

Manuscript received August 20, 2013. The associate editor coordinating the review of this letter and approving it for publication was J. Tugnait.

The authors are with the Department of Electrical Engineering, Southern Methodist University, Dallas, TX, 75206 USA (e-mail: \{phuang, rajand, camp\}@smu.edu).

This material is based upon work supported by the National Science Foundation under grant numbers: 0958436, 1040429, and 1150215.

Digital Object Identifier 10.1109/WCL.2013.091113.130593 method has two main characteristics. First, we use a damped sinusoid to approximate the autocorrelation of the Rayleigh fading channel. Second, we utilize an order-two AR model to describe the damped sinusoid. The maximum Doppler value is then evaluated as a function of the coefficients of the AR model. Numerical results demonstrate the superiority of the proposed method over existing state-of-the-art methods.

The remainder of this paper is organized as follows. In Section II, the similarity between the first-kind, order-zero Bessel function and the damping sinusoid is analyzed. Section III presents the novel Doppler spread estimation scheme. In Section IV, numeric simulation results are demonstrated, and conclusions are drawn in Section V.

\section{ApProximated First-KIND, ORDER-ZERo BESSEL (BESSEL $(1,0))$ FunCTION By DAMPED SinUSOID}

In this paper, we model the fading channel, $h(t)$, using the popular Jakes' model [11]. According to this model, the arrival angles $\alpha_{l}(l=1,2, \ldots, L)$ of the $L$ multiple paths are uniformly distributed over $[0, \pi]$. Under this condition of uniformly distributed arrival angle, the autocorrelation, $R(\tau)$, of the Rayleigh fading channel $h(t)$ can be shown to equal the zeroth order Bessel function of the first kind, $J_{0}(\cdot)$, as

$$
R(\tau)=\frac{1}{L} J_{0}\left(2 \pi f_{D} \tau\right)
$$

where $f_{D}$ is the maximum Doppler spread. Since, the autocorrelation function $R(\tau)$ contains information about the Doppler spread, we first evaluate the autocorrelation and then develop an estimator for the Doppler spread. The complexity of the Bessel function, however, makes it challenging to develop an efficient estimator. Consequently, we approximate the Bessel function with a damped sinusoid.

To demonstrate the accuracy of the approximation, we recall some characteristics of the Bessel function. It is well known that the zeroth order Bessel function of the first kind is the solution of the following differential equation:

$$
t^{2} \frac{d^{2} x(t)}{d t^{2}}+t \frac{d x(t)}{d t}+t^{2} x(t)=0 .
$$

The periodicity of the Bessel function asymptotically reaches $\pi$ as $t \rightarrow \infty$. Now, we consider the behavior of a spring system, which is modeled using the following differential equation:

$$
\frac{d^{2} x(t)}{d t^{2}}+\frac{c}{m} \frac{d x(t)}{d t}+\frac{k}{m} x(t)=0,
$$

where $m$ is the mass of the ball attached to the spring, $k$ is the elastic coefficient of the spring, and $c$ is damping factor in the system. This spring system is in the underdamped condition when

$$
\left(\frac{c}{m}\right)^{2}-\frac{4 k}{m}<0
$$


The solution to (3) is given by a damped sinusoid of the form,

$$
X_{D S}(t)=a^{-t} \cos \left(\omega_{0} t+\phi_{0}\right) .
$$

Here, the damping factor is $a=e^{\frac{c}{2 m}}$, the radian frequency is $\omega_{0}=\frac{\sqrt{4 m k-c^{2}}}{2 m}$, and $\phi_{0}$ denotes the initial phase of the damping sinusoid, which is determined by the initial position of the oscillator. Further, the time period $T_{D S}$ of the oscillator is given by:

$$
T_{D S}=\frac{2 \pi}{\omega_{0}}=\frac{2 \pi}{\frac{\sqrt{4 m k-c^{2}}}{2 m}} .
$$

Now, consider the case that the elastic coefficient $(k)$ of the spring equals to the mass $(m)$ of the oscillator $(k=m)$ and the damping factor $\left(\left.c \rightarrow 0\right|_{t \rightarrow \infty}\right)$. In this case, the time period $T_{D S}$ of the damped sinusoid equals the asymptotic time period of the Bessel function. Thus, the differential equation whose solution equals the Bessel function can be regarded as a virtual spring oscillator system which has a time-varying damping force. Fig. 1 illustrates the damping sinusoid $\left(x_{1}(t)\right)$ and the Bessel $(1,0)$ function $\left(x_{2}(t)\right)$. From Fig. 1, we can see the zero-crossing periods of the two curves tend to become identical as time $t$ increases. Thus, we can acquire the Doppler information from the zero-crossing period of the damping sinusoid.

\section{MAXIMUM DOPPLER ESTIMATION BASED ON DAMPED SINUSOID OSCILLATOR}

In the previous section, we presented an approximation for the Bessel $(1,0)$ function using a damped sinusoid and illustrated the reasonability of the approximation. In this section, we introduce a novel Doppler estimation scheme and discuss its application in an OFDM system. First, we calculate the autocorrelation of the received signal in baseband, which contains information about the maximum Doppler. Second, we use the damping sinusoid to approximate the Bessel $(1,0)$ function and then transform the damped sinusoid using an order-two AR model. Third, we compute the coefficients of the AR model by solving the Yule-Walker equation. Finally, we calculate the maximum Doppler spread based on the coefficients of the AR model.

\section{A. AR model based description of damping sinusoid}

A sinusoid can be described recursively by an order-two AR model as follows:

$$
\begin{gathered}
\cos (n \omega)=2 \cos \omega \cos (n-1) \omega-\cos (n-2) \omega \\
\sin (n \omega)=2 \cos \omega \sin (n-1) \omega-\sin (n-2) \omega .
\end{gathered}
$$

Similarly, a damped sinusoid can be expressed as:

$e^{n \lambda} \cos (n \omega)=2 e^{-\lambda} \cos \omega \cos ((n-1) \omega)-e^{-2 \lambda} \cos ((n-2) \omega)$.

From (8), the coefficient $2 e^{-\lambda} \cos \omega$ contains the Doppler information $(\omega)$, which will be utilized in the estimation scheme.

\section{B. Maximum Doppler estimation scheme}

Since the autocorrelation of the fading channel is being approximated by the damped sinusoid, the autocorrelation can be written in recursive form as:

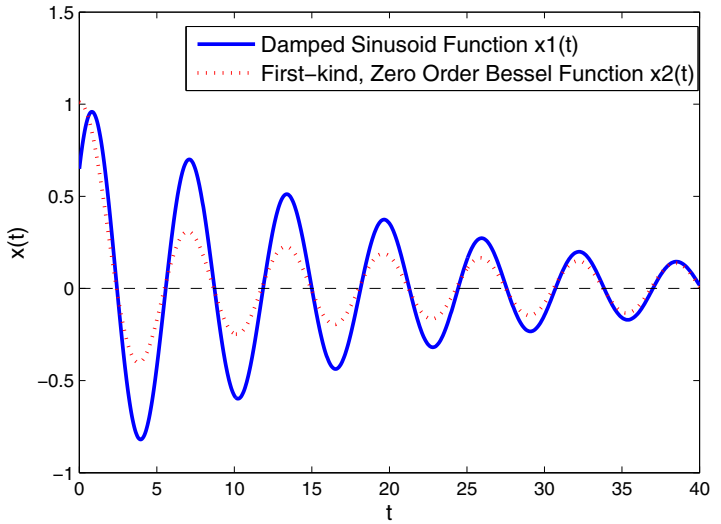

Fig. 1. Contrast between Bessel $(1,0)$ curve and damping sinusoid

$$
R(n)=2 e^{-\lambda} \cos \left(2 \pi f_{D}\right) R(n-1)-e^{-2 \lambda} R(n-2) .
$$

If $N$ samples, $R(0), R(1), \ldots, R(N-1)$, of the fading channel autocorrelation are available, the Yule-Walker equations are given by:

$$
\left[\begin{array}{cc}
R(0) & R(1) \\
R(1) & R(2) \\
\vdots & \vdots \\
R(N-3) & R(N-2)
\end{array}\right]\left[\begin{array}{l}
b_{1} \\
b_{2}
\end{array}\right]=\left[\begin{array}{c}
R(2) \\
R(3) \\
\vdots \\
R(N-1)
\end{array}\right]
$$

where $b_{1}=2 e^{-\lambda} \cos \omega, b_{2}=-e^{-2 \lambda}$. From (10), the coefficients $\hat{\mathbf{b}}=\left[\hat{b}_{1}, \hat{b}_{2}\right]^{T}$ of the order-two AR model can be calculated as:

$$
\hat{\mathbf{b}}=\left(\mathbf{R}_{\mathbf{M}}^{\mathbf{T}} \mathbf{R}_{\mathbf{M}}\right)^{-\mathbf{1}} \mathbf{R}_{\mathbf{M}}^{\mathbf{T}} \mathbf{R}_{\mathbf{V}},
$$

where, $\quad \mathbf{R}_{\mathbf{M}}=\left[\begin{array}{llll}R(0) & R(1) & \ldots & R(N-3) \\ R(1) & R(2) & \ldots & R(N-2)\end{array}\right]^{\mathbf{T}}$ and $\mathbf{R}_{\mathbf{V}}=\left[\begin{array}{llll}R(2) & R(3) & \ldots & R(N-1)\end{array}\right]^{\mathbf{T}}$.

With the estimated coefficients $\left[\hat{b}_{1}, \hat{b}_{2}\right]$, the maximum Doppler spread, $f$, can be calculated as:

$$
\hat{f}_{D}=\frac{1}{2 \pi} \arccos \left(\frac{\hat{b}_{1}}{2 \sqrt{-\hat{b}_{2}}}\right) \text {. }
$$

We now analyze the bias and the variance of $f_{D}$. From [12], it is known that the estimate of the AR model coefficients $\left[\hat{b}_{1}, \hat{b}_{2}\right]$ are asymptotically unbiased. Further, this estimate can be written as [13]

$$
\hat{b}_{i}=b_{i}+\Delta b_{i}
$$

where $\Delta b_{i}(i=1,2)$ denotes the estimation bias. Based on [12], the mean of the bias $\left(\operatorname{mean}\left(\Delta b_{i}\right)\right)$ and the variance $\operatorname{var}\left(\Delta b_{i}\right)$ vary asymptotically as $O\left(\frac{1}{N}\right)$. Substituting (13) into (12) and after simple algebraic analysis, we obtain

$$
\hat{f}_{D} \approx f_{D}\left(1-\frac{\Delta b_{1}}{4 \pi f_{D}}\right)
$$

Thus, the bias and the variance of the proposed maximum Doppler estimator asymptotically approach 0 as $N$ tends to infinity. 


\section{Application to OFDM Systems}

We now discuss the application of the proposed Doppler estimation algorithm to an OFDM system.

The transmitted signal, $s(n)$, at time $n$ in an OFDM system can be written as:

$$
s(n)=\sqrt{\frac{1}{I}} \sum_{k=0}^{P-1} d_{k} e^{j 2 \pi n k / I} .
$$

Here, $d_{k}$ is the information modulated on the k-th subcarrier and is assumed to be zero mean, $I$ is the total number of subcarriers and $P \leq I$ is the number of subcarriers carrying a known pilot signal.

The fading channel at time $n$ has $L+1$ taps and is represented by $h(n, l), 0 \leq l \leq L$. Thus, the received signal $y(n)$ can be written as:

$$
y(n)=\sum_{l=0}^{L} h(n, l) s(n-l)+\xi(n),
$$

where $\xi(n)$ denotes the additive noise in the system and $h(n, l)$ has zero mean [14]. Combining (15) and (16), we can rewrite the received OFDM signal, $y(n)$, as:

$$
y(n)=\frac{1}{\sqrt{I}} \sum_{k=0}^{P-1} d_{k} H_{k}(n) e^{j 2 \pi n k / I}+\xi(n),
$$

where $H_{k}(n)=\sum_{l=0}^{L} h(n, l) e^{-j 2 \pi l k / I}$.

To estimate Doppler, the autocorrelation $R_{y}$, of the received signal is calculated as:

$$
\begin{aligned}
R_{y}\left(n_{1}-n_{2}\right) & \\
= & E\left[y\left(n_{1}\right) y^{*}\left(n_{2}\right)\right] \\
=E & {\left[\left(\frac{1}{\sqrt{I}} \sum_{k=0}^{P-1} d_{k} H_{k}\left(n_{1}\right) e^{j 2 \pi n_{1} k / I}+\xi\left(n_{1}\right)\right)\right.} \\
\cdot & \left.\left(\frac{1}{\sqrt{I}} \sum_{p=0}^{P-1} d_{p}^{*} H_{p}^{*}\left(n_{2}\right) e^{-j 2 \pi n_{2} p / I}+\xi\left(n_{2}\right)\right)\right] \\
=E & {\left[\frac{1}{I} \sum_{k=0}^{P-1} \sum_{p=0}^{P-1} d_{k} d_{p}^{*}\right.} \\
& \left.\cdot H_{k}\left(n_{1}\right) H_{p}^{*}\left(n_{2}\right) e^{j 2 \pi n_{1} k / I} e^{-j 2 \pi n_{2} p / I}\right] \\
+ & \delta_{\xi}\left(n_{1}-n_{2}\right),
\end{aligned}
$$

where $\delta_{\xi}\left(n_{1}-n_{2}\right)=E\left[\xi\left(n_{1}\right) \xi^{*}\left(n_{2}\right)\right]$ and the cross terms involving $\xi$ equals zero since the noise is assumed to be zero mean. Now, the autocorrelation can be rewritten as,

$$
\begin{aligned}
& R_{y}\left(n_{1}-n_{2}\right) \\
& =E\left[\frac{1}{I} \sum_{k=0}^{P-1}\left|d_{k}\right|^{2} H_{k}\left(n_{1}\right) H_{k}^{*}\left(n_{2}\right) e^{j 2 \pi\left(n_{1}-n_{2}\right) k / I}\right] \\
& +E\left[\frac{1}{I} \sum_{k=0, k \neq p}^{P-1} \sum_{p=0}^{P-1} d_{k} d_{p}^{*}\right. \\
& \left.\qquad H_{k}\left(n_{1}\right) H_{p}^{*}\left(n_{2}\right) e^{j 2 \pi n_{1} k / I} e^{-j 2 \pi n_{2} p / I}\right] \\
& +\delta_{\xi}\left(n_{1}-n_{2}\right) .
\end{aligned}
$$

We assume that the pilot symbols $d_{k}$ are selected to have a uniform amplitude. This assumption is not criticial and is only used to simplify the final expression. Consequently, we can rewrite (19) as:

$$
\begin{aligned}
R_{y}( & \left.n_{1}-n_{2}\right) \\
= & E\left[\frac{1}{I} \sum_{k=0}^{P-1}\left|d_{k}\right|^{2} H_{k}\left(n_{1}\right) H_{k}^{*}\left(n_{2}\right) e^{j 2 \pi\left(n_{1}-n_{2}\right) k / I}\right] \\
& +\delta_{\xi}\left(n_{1}-n_{2}\right) \\
= & \sum_{k=0}^{P-1} \frac{1}{I}\left|d_{k}\right|^{2} e^{j 2 \pi\left(n_{1}-n_{2}\right) k / I} \\
& \cdot E\left[\sum_{l=0}^{L} h\left(n_{1}, l\right) e^{-j 2 \pi l k / I} \sum_{m=0}^{L} h\left(n_{2}, m\right) e^{j 2 \pi m k / I}\right] \\
& +\delta_{\xi}\left(n_{1}-n_{2}\right) \\
= & \sum_{k=0}^{P-1} \frac{L}{I}\left|d_{k}\right|^{2} e^{j 2 \pi q k / I} J_{0}\left(2 \pi f_{D} q\right)+\delta_{\xi}(q),
\end{aligned}
$$

where $q=n_{1}-n_{2} ; E\left[h\left(n_{1}, l\right) h\left(n_{2}, m\right)\right]=J_{0}\left(2 \pi f_{D} q\right)$ when $l=m$ [11] and $E\left[h\left(n_{1}, l\right) h\left(n_{2}, m\right)\right]=0$ when $l \neq m$. Thus, $R_{y}(q)$ can be further simplified according to:

$$
R_{y}(q)=\frac{L}{I} J_{0}\left(2 \pi q f_{D}\right) \frac{e^{j 2 \pi q P / I}-1}{e^{j 2 \pi q / I}-1}+\delta_{\xi}(q) .
$$

Since the value of $P$ is fixed and the values of parameters $q$ and $I$ are known, we can estimate the maximum Doppler value from the computed autocorrelation result using the proposed method. Algorithm 1 describes the main steps in the proposed estimation method.

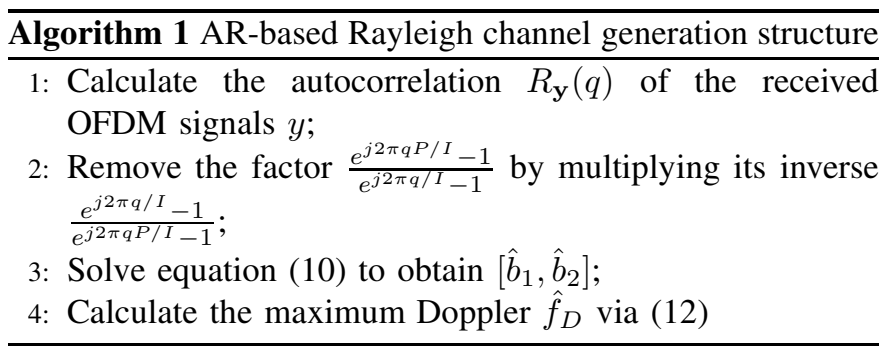

\section{Numeric Simulations AND ANAlysis}

In this section we present simulation results to illustrate the performance of the proposed estimation scheme. We consider an OFDM system with 64 subcarriers $(P=64)$ of the pilot signal and the fading channel has 6 taps $(L=6)$ which refers to the ITU Vehicular Channel B model.

Fig. 2 plots the RMSE of the estimated Doppler value as a function of SNR. The normalized Doppler is 0.1, which is the product between the actual Doppler and the symbol duration, and the results are averaged over $10^{2}$ independent realizations of the experiments. For comparison, the RMSE using existing Doppler estimators from [2], [3], [5], [6] are also shown. And the estimation performance without a pilot signal is also considered in this experiment. The ROM stands for rate of maxima. Generally, the ROM based method implement the Doppler estimation by counting the number of the maxima 


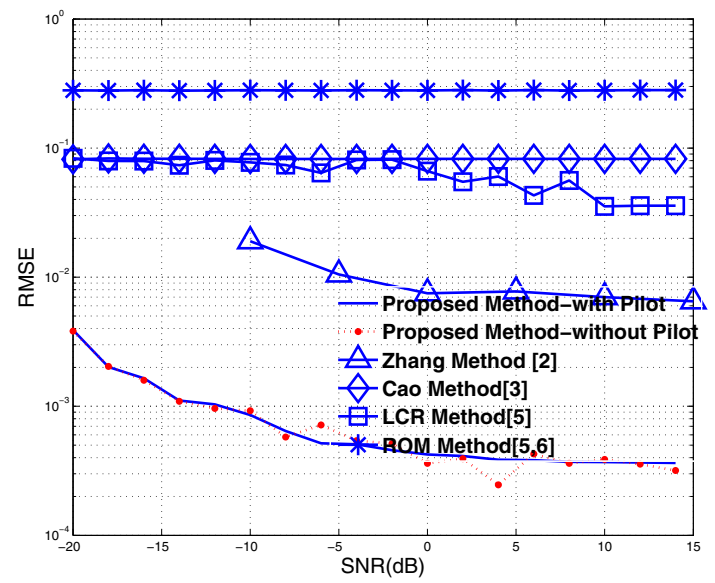

Fig. 2. RMSE of estimated Doppler values versus SNR.

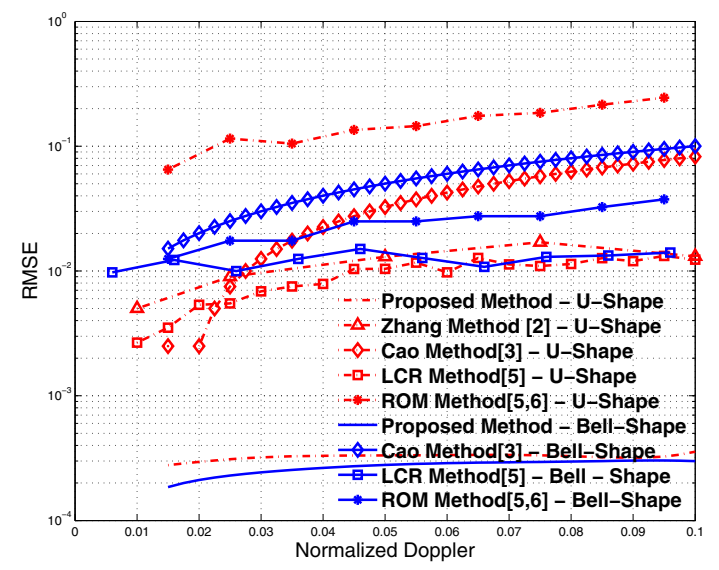

Fig. 3. RMSE of estimated Doppler values for different Doppler spectrum.

points in the channel gain autocorrelation. And the LCR method is based on the Level Crossing Rate of the fading channel. The results clearly show the significant reduction in RMSE of the proposed method.

In Fig. 3, the RMSE is plotted as a function of the normalized Doppler at an SNR of $5 \mathrm{~dB}$. To illustrate the widespread applicability of the proposed method, we also show the results with a 'Bell' shaped Doppler spectrum. From Fig. 3, the superior performance of the proposed scheme over existing methods in [2], [3], [5], [6] is evident.

\section{CONCLUSIONS}

In this paper, we propose a novel Doppler spread estimator which utilizes a damping sinusoid to approximate the autocorrelation of the fast-fading channel. The proposed Doppler spread estimator has low complexity and high accuracy and is appropriate for implementation in practical systems such as vehicular networks.

\section{REFERENCES}

[1] Y. Tsai and K. Yang, "Approximate ML Doppler spread estimation over flat Rayleigh fading channels," IEEE Signal Process. Lett., vol. 16, no. 11, pp. 1007-1010, Nov. 2009.

[2] H. Zhang and A. Abdi, "Cyclostationarity-based Doppler spread estimation in mobile fading channels," IEEE Trans. Commun., vol. 57, no. 4, pp. 1061-1067, Apr. 2009.

[3] M. Cao, H. Ge, H. Zhang, and A. Abdi, "Parametric Doppler spread estimation in mobile fading channels," in Proc. 2007 IEEE Military Communications Conference, pp. 1-5.

[4] C. Tepedelenlioglu, A. Abdi, G. Giannakis, and M. Kaveh, "Estimation of Doppler spread and signal strength in mobile communications with applications to handoff and adaptive transmission," Wireless Commun. and Mobile Computing, vol. 1, no. 2, pp. 221-242, 2009.

[5] G. L. Stüber, Principles of Mobile Communication. Kluwer Academic Publishers, 2001.

[6] A. Abdi, H. Zhang and C. Tepedelenlioglu, "A unified approach to the performance analysis of speed estimation techniques in mobile communication," IEEE Trans. Commun., vol. 56, no. 1, pp. 126-135, 2008.

[7] K. E. Baddour and N. C. Beaulieu, "Autoregressive modeling for fading channel simulation," IEEE Trans. Wireless Commun., vol. 4, no. 4, pp. 1650-1662, Jul. 2005.

[8] K. Muralidhar, K. H. Li, and K. V. S. Hari, "Iterative Kalman-AR method for Doppler spread estimation in flat fading channels," in Proc. 2007 IEEE International Symposium on Personal, Indoor and Mobile Radio Communications, pp. 1-5.

[9] M. Souden, S. Affes, J. Benesty, and R. Bahroun, "Robust Doppler spread estimation in the presence of a residual carrier frequency offset," IEEE Trans. Signal Process., vol. 57, no. 10, pp. 4148-4153, 2009.

[10] T. Yucek, R. M. A. Tannious, and H. Arslan, "Doppler spread estimation for wireless OFDM systems," in Proc. 2005 IEEE/Sarnoff Symposium on Advances in Wired and Wireless Communication, pp. 233-236.

[11] W. Jake, Microwave Mobile Communication. Wiley-IEEE Press, 1974.

[12] S.M. Kay, Modern Spectral Estimation: Theory and Application. Prentice Hall, 1988.

[13] B.C. Levy, Principles of Signal Detection and Parameter Estimation. Springer US, 2008.

[14] J. K. Tugnait, S. He, and H. Kim, "Doubly selective channel estimation using exponential basis models and subblock tracking," IEEE Trans. Signal Process., vol. 58, no. 3, pp. 1275-1289, Mar. 2010. 